\section{References}

Hounsfield, G. N. (1973) Computerised transverse axial scanning (tomography): Part I. Description of a system. British Journal of Radiology, 46, 1016-1022.

OWEns, D. G. C., Johnstone, E. C., BYdDer, G. M. \& KREEL, L. (1980) Unsuspected organic disease in chronic schizophrenia demonstrated by computed tomography. Journal of Neurology. Neurosurgery and Psychiatry, 43, 1065-1069.

RoberTs, J. K. A. \& Lishman, W. A. (1984) The use of the CAT head scanner in clinical psychiatry. British Journal of Psychiatry, 145, 152-158.

\title{
Development of outcome measures in acute psychiatry
}

\author{
Femi Oyebode, Consultant Psychiatrist; Stuart Cumella, Senior Research Fellow, \\ Centre for Research and Information into Mental Disability; GILL GARDEN, Senior \\ Registrar; and JUDITH Nicholls, Senior Registrar, Queen Elizabeth Psychiatric \\ Hospital, Edgbaston, Birmingham B15 2QZ
}

This study is an evaluation of the use of two health status measures for monitoring the outcome of acute psychiatric treatment. The measures are the Sickness Impact Profile (SIP) and the Classification of Illness States (CIS). The SIP is a measure of the impact of sickness on patients' daily activities and behaviour. The CIS was initially developed to measure 'morbidity states' after hospital treatment and can be used to generate the 'Rosser Index', both of which have been shown to be valid and reliable measures (Rosser, 1988).

The aim of this investigation was to determine whether these health status measures were applicable in psychiatry. We also examined the ease of administering the questionnaires and whether sample survey of patients is practicable up to one year after discharge from hospital.

\section{The study}

All patients discharged from hospital in the adult psychiatric service between 1 April 1988 and 31 March 1989, resident at the time of their admission in two selected electoral wards of Birmingham, were eligible for inclusion. Two separate clinical teams were responsible for the wards which were selected to ensure inclusion of patients from a wide range of ethnic groups and domestic circumstances. Electoral ward ' $A$ ' had a population of 22,800 in 1981 and the Jarman index was 26.9. Electoral ward 'B' had a population of 24,400 in 1981 and the Jarman index was 1.5.
Patients who agreed to be interviewed were visited at home or in hospital or seen in out-patient clinics by a psychiatrist. Where there was no formal refusal to participate, a minimum of three visits were made to the home addresses before the patient was regarded as uncontactable. All patients who were seen completed the SIP, CIS and Hospital Anxiety and Depression (HAD) Scale. A short questionnaire with questions on type of housing, constitution of household, and employment circumstances was administered. Data on personal characteristics, diagnoses, and admission history of the total sample were obtained from the Unit Case Register. The interviews were conducted between April and September 1990.

\section{Findings}

One hundred and two patients were eligible for inclusion of whom 61 were contactable. Eleven patients refused to participate. Three were too cognitively impaired to complete the questionnaires and one patient had died. There was a lower response rate in Ward 'A' (18/51) compared with Ward 'B' $(29 / 51)$.

Of the 51 respondents, 38 were living in private households, six in hostels, and four in psychiatric hospital wards. In all, 29 patients had re-admissions in the year following the index discharge. Ten of the respondents were working at follow-up, one was in full-time education, and three were providing fulltime care for their families. The remainder included 
six patients in the labour market but unemployed, 10 retired and 18 who said they were too sick to look for work.

The SIP scores were highest for the scales relating to the impact of ill-health upon personal behaviour (alertness, emotional behaviour and social interaction), and on general social roles (recreation and pastimes, work and household management). On the CIS, the majority of patients had 'moderate' (12) or 'severe' (18) levels of distress. Eighteen had 'no disability' or 'slight social disability', and a further 15 'severe social disability and/or slight impairment of performance at work'. The remaining 14 patients had 'severe disabilities', including one who was confined to bed.

The mean scores for both HAD scales were below the case threshold of $10 / 11$, and 29 of the 47 patients who completed the HAD had scores below threshold for both the anxiety and depression scales. The remaining 18 included eight cases on the anxiety scale alone, two on the depression scale alone, and eight on both scales.

Only two variables emerged as predictors of outcome. Patients with neuroses and adjustment reactions were most likely to have high scores on the SIP Physical Dimension and age correlated positively to the SIP Physical Dimension.

Patients experienced few problems completing either the HAD or CIS questionnaires; each took no more than 10 minutes. However, the SIP usually took 30-90 minutes to complete, and the psychiatrist often had to explain questions to patients.

\section{Comment}

The CIS questionnaire was easy to administer. The CIS scales have a limited range of ratings and produced a narrow range of scores. This confirms earlier research with psychiatric patients (Wilkinson et al, 1990). The CIS and associated questionnaire may not be appropriate for routine audit purposes as it may not be sensitive enough to detect changes in quality of life, especially in chronic psychiatric patients. Nevertheless, the Rosser Index represents an important step forward in the measurement of health status, and further research into its application in psychiatry is recommended. Its application as a basis for calculating quality-adjusted life years (QALYs) has been widely publicised. QALYs, it is argued, will provide a more rational basis for making resource allocation decisions (Gudex, 1986). The conceptual and methodological problems with QALYs are many: there is an emphasis on extension of life which may not be appropriate for psychiatry; often information about outcome on which QALYs is calculated is inadequate; and, the valuation of illness states was based upon responses from a relatively small sample of 70 subjects (Gudex, 1986; Wilkinson et al, 1990).

The SIP has the advantage of assessing the impact of the patient's health on several items relating to general social functioning. It can therefore be used to evaluate the impact of treatment on such factors as recreation, housework or employment. However, it is cumbersome to administer. It would probably be more appropriate in sample surveys, and in assessing the impact of ill health on patients with chronic disorders.

The HAD was used as an independent measure of psychiatric morbidity. It was easy to administer and it correlated well with the SIP. However, it has a narrower focus as it does not (nor was it meant to) measure general health status or social functioning.

The low response rate in this follow-up survey especially in the electoral ward with a high Jarman score underlined the difficulty of tracing patients in inner city areas where the population is highly mobile. This suggests that outcome surveys should be instituted closer to the date of discharge.

\section{References}

Gudex, C. (1986) QALYs and Their Use by the Health Service. Discussion paper 20. York: University of York Centre for Health Economics.

Rosser, R. M. (1988) A health index and output measure. In Quality of Life: assessment and application (edited S. R. Walker and R. M. Rosser). Lancaster: MTP.

Wilkinson, G., Croft-Jefrreys, C., Krekorian, H. et al (1990) QALYs in psychiatric care. Psychiatric Bulletin. $14,582-585$.

A full list of references is available from Dr Oyebode. 